\title{
Effects of Inertia on the Steady-Shear Rheology of Disordered Solids
}

\author{
Alexandre Nicolas ${ }^{*}$ and Jean-Louis Barrat \\ LIPhy, Université Grenoble-Alpes \& CNRS, F-38000 Grenoble, France \\ Jörg Rottler \\ Department of Physics and Astronomy, The University of British Columbia, \\ 6224 Agricultural Road, Vancouver, British Columbia V6T 1Z4, Canada
}

(Received 26 August 2015; revised manuscript received 29 October 2015; published 5 February 2016)

\begin{abstract}
We study the finite-shear-rate rheology of disordered solids by means of molecular dynamics simulations in two dimensions. By systematically varying the damping strength $\zeta$ in the low-temperature limit, we identify two well-defined flow regimes, separated by a thin (temperature-dependent) crossover region. In the overdamped regime, the athermal rheology is governed by the competition between elastic forces and viscous forces, whose ratio gives the Weissenberg number Wi $\propto \zeta \dot{\gamma}$; the macroscopic stress $\Sigma$ follows the frequently encountered Herschel-Bulkley law $\Sigma=\Sigma_{0}+k \sqrt{\mathrm{Wi}}$, with yield stress $\Sigma_{0}>0$. In the underdamped (inertial) regime, dramatic changes in the rheology are observed for low damping: the flow curve becomes nonmonotonic. This change is not caused by longer-lived correlations in the particle dynamics at lower damping; instead, for weak dissipation, the sample heats up considerably due to, and in proportion to, the driving. By thermostating more or less underdamped systems, we are able to link quantitatively the rheology to the kinetic temperature and the shear rate, rescaled with Einstein's vibration frequency.
\end{abstract}

DOI: 10.1103/PhysRevLett.116.058303

Inertia matters in liquid flows. Its presence in the NavierStokes equations leads to a rich phenomenology that vanishes in the overdamped limit of viscous flow. However, the effect of damping is rarely heeded (let alone analyzed) in the flow of disordered solids, so much so that dense colloidal glasses often serve as model systems for bulk metallic glasses (BMG) [1], even though they are much more strongly damped. Here, we find that reducing the damping can dramatically impact the macroscopic rheology. In the inertial regime, the energy input dwells longer in the particle momenta before its final dissipation into the heat bath, thus facilitating plastic flow. We provide a quantitative account of this effect in terms of simple kinetic heating of the underdamped solid, similar to the one observed experimentally during the operation of shear bands in BMG [2,3].

The damping regime is not the only line of contrast among disordered solids: atoms in BMG as well as small colloids are heavily influenced by thermal fluctuations whereas grains are quasiathermal; foam bubbles are deformable whereas some colloids are close to perfect hard spheres. Notwithstanding these contrasting features, virtually all such solids deform similarly, i.e., mostly elastically at small stresses while at larger shear plasticity becomes dominant, with a succession of failures of microregions, whose particles rearrange swiftly. These rearrangements are triggered by the loading or facilitated by thermal activation [4,5] and may interact via the long-range elastic deformation that they induce in the surrounding medium
$[5,6]$. Based on this generic scenario, multiple simplified rheological models have been proposed, generally focusing on the overdamped regime [7-12] (nevertheless, the mesoscale elastic response has been studied across the damping regimes $[13,14])$. To what extent does the presence of inertia alter the picture?

In the quasistatic limit, i.e., at vanishing shear rates $\dot{\gamma}$, recent numerical work by Salerno and Robbins has ascertained that the statistics of avalanches fall into distinct universality classes in the overdamped vs underdamped regimes $[15,16]$. The difference is best illustrated by considering the complex, rugged potential energy landscape (PEL) in which the system evolves: it climbs up energy barriers in the phases of elastic loading and abruptly slides downhill once the barrier is overcome. For overdamped systems, this descent suffices to dissipate the energy stored during loading, while at lower damping the inertial force may carry the system over several successive barriers. This process is then highly directional in the PEL and strongly correlated in space and time, which renders its modeling quite complex a priori. Some ad hoc rules to include inertia in lattice-based models have been put forward, such as lowering barriers or yield stresses for a certain time after failure (see Ref. [17] and references in [16]) and their impact has been emphasized, but the validity of these descriptions stands on shaky ground.

In this Letter, we focus on the steady-state shear flow of two-dimensional disordered solids at finite driving rates and investigate the role of inertia in the vanishing and low 
temperature limits, with molecular dynamics (MD) simulations. We apply simple shear to the binary Lennard-Jones glass used in Ref. [14]; it comprises 32500 large (type $A$ ) particles and 17500 small (type $B$ ) particles, all of mass $m$, and has reduced density $\rho=1.2$. The equations of motion are based on the dissipative particle dynamics (DPD) scheme [18] and read

$$
\begin{aligned}
\frac{d \mathbf{r}_{\mathbf{i}}}{d t} & =\mathbf{v}_{\mathbf{i}} \\
m \frac{d \mathbf{v}_{\mathbf{i}}}{d t} & =-\sum_{i \neq j} \frac{\partial \mathcal{V}\left(r_{i j}\right)}{\partial \mathbf{r}_{\mathbf{i j}}}+f_{i}{ }^{R}+\boldsymbol{f}_{i}{ }^{D} .
\end{aligned}
$$

Here, $\boldsymbol{r}_{i j} \equiv \boldsymbol{r}_{\boldsymbol{i}}-\boldsymbol{r}_{j}$ and $\mathcal{V}\left(r_{i j}\right)$ is the interaction potential between particles $i$ and $j$. The DPD forces $f_{i}{ }^{R, D}$ involve a cutoff function $w(r) \equiv 1-\left(r / r_{c}\right)$ if $r<r_{c} \equiv 2.5 \sigma_{A A}$ and 0 otherwise; $f_{i}{ }^{R} \equiv s \sum_{j \neq i} w\left(r_{i j}\right) \theta_{i j}\left(\boldsymbol{r}_{i j} / r_{i j}\right)$ is a stochastic force, based on the Gaussian white noise $\theta_{i j}$ [18] and due to the coupling to a heat bath maintained at temperature $T_{0}$ and $\boldsymbol{f}_{i}{ }^{D} \equiv-\zeta \sum_{j \neq i} w^{2}\left(r_{i j}\right)\left(\boldsymbol{v}_{i j} \cdot \boldsymbol{r}_{i j} / r_{i j}^{2}\right) \boldsymbol{r}_{i j}$ is a damping force depending on the relative velocities $v_{i j} \equiv \boldsymbol{v}_{\boldsymbol{i}}-\boldsymbol{v}_{j}$. The strength $s=2 \zeta k_{B} T_{0}$ of the coupling to the reservoir depends on the damping strength $\zeta$ and $T_{0}$, and is maintained even if the system departs from thermal equilibrium. In the following, $\zeta, m$, and $T_{0}$ shall be varied, while the particle interactions are kept constant.

The equations of motion, Eqs. (1), are integrated on GPU with the velocity Verlet algorithm. They involve forces deriving from four types of stresses:

(i) the elastic stress, of order $\Sigma_{A} \equiv \epsilon_{A A} / \sigma_{A A}^{2} \equiv 1$,

(ii) the viscous stress, of order $\eta \dot{\gamma}$, where $\eta \approx \zeta[14]$ is the microscopic viscosity,

(iii) the inertial pressure, which, in a Bagnold-like picture [19], involves momentum transfers of order $m \sigma_{A A} \dot{\gamma}$ at a rate $\propto \dot{\gamma}$, and is thus proportional to $m \dot{\gamma}^{2}$, and

(iv) the thermal pressure resulting from stochastic forces of magnitude $\sqrt{\zeta T_{0}}$.

Their relative magnitudes are quantified by dimensionless numbers that characterize the flow regime. In particular, the importance of viscosity with respect to elasticity is measured by the Weissenberg number,

$$
\mathrm{Wi} \equiv \tau_{\text {diss }} \dot{\gamma} \quad \text { with } \quad \tau_{\text {diss }} \equiv \frac{\zeta}{\Sigma_{A}}
$$

and the ratio of inertial over elastic stresses is given by $\mathrm{Ei}^{2}$, where

$$
\mathrm{Ei} \equiv \tau_{\mathrm{vib}} \dot{\gamma} \quad \text { with } \quad \tau_{\mathrm{vib}} \equiv \sqrt{\frac{m}{\Sigma_{A}}}
$$

In conjunction with $T_{0}, \mathrm{Wi}$ and Ei fully characterize the flow. Nevertheless, to describe the damping regime of flow curves, irrespective of the shear rate, it is convenient to also introduce

$$
Q \equiv \frac{\mathrm{Ei}}{\mathrm{Wi}}=\frac{\sqrt{m \Sigma_{A}}}{\zeta}=\frac{\tau_{\mathrm{damp}}}{\tau_{\mathrm{vib}}} \quad \text { with } \quad \tau_{\mathrm{damp}} \equiv \frac{m}{\zeta}
$$

if Eq. (1) is assimilated to a damped second-order harmonic oscillator, $Q$ is the (inertial) quality factor, i.e., the number of inertial oscillations in the damping time.

Our numerical data confirm the relevance of such dimensional analysis: Figures 1 and 2 prove that, at $T_{0}=0$, the dependences of the macroscopic shear stress $\Sigma$ on $\zeta, m$, and $\dot{\gamma}$ can be condensed into a dependence on the pair $(Q, \mathrm{Wi})$, or equivalently (but more conveniently when $Q \gg 1)(Q, \mathrm{Ei})$.

Overdamped dynamics.- - Let us start by investigating the fully overdamped (Brownian or athermal) limit $Q \rightarrow 0$. In the absence of inertia, $\dot{\gamma}$ is best rescaled as Wi. At $T_{0}=0$, the flow curve, plotted in Fig. 1, is very well described by the Herschel-Bulkley law

$$
\Sigma\left(\mathrm{Wi}, T_{0}=0\right)=0.72+2 \sqrt{\mathrm{Wi}} .
$$

Interestingly, this description remains very good at finite values of $Q$, up to $Q \approx 1$. Thus, for all $Q \leq 1$, the macroscopic rheology is exclusively governed by the competition between elastic and viscous forces.

Leaving the athermal regime, we observe that imposing a finite bath temperature $T_{0}>0$ leads to a decrease of $\Sigma$ at all shear rates. Regardless of the damping regime, this thermal effect is explained by the premature occurrence of plastic rearrangements owing to thermal activation: the system at $T_{0}>0$ hops over saddle-node points in the PEL before the effective potential barriers have completely flattened under the influence of the driving, which interrupts the elastic accumulation of strain, hence, the lower macroscopic stress $[20,21]$.

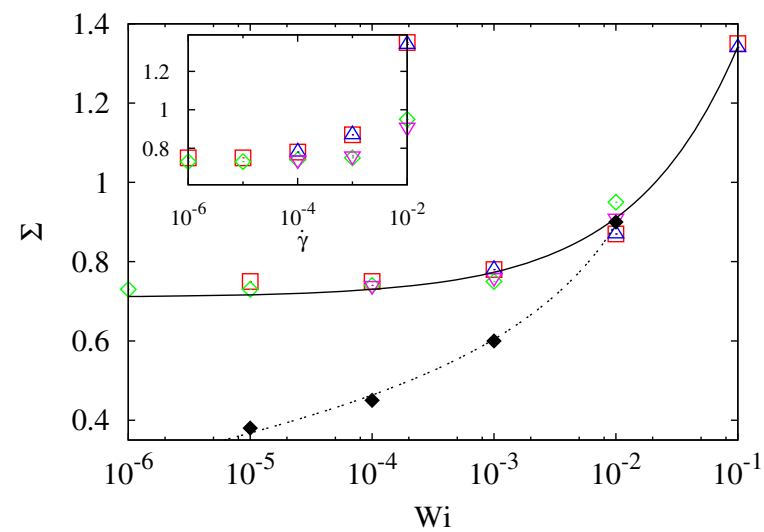

FIG. 1. Athermal flow curves $\Sigma\left(\mathrm{Wi}, T_{0}=0\right)$ in the overdamped regime $Q \lesssim 1$, for various combinations $[\zeta, m]:[\zeta=$ $1, m=1](\diamond),[10,1](\square),[10,0.1](\triangle)$, and $[1,0.1](\nabla)$. The solid line represents Eq. (2). A flow curve at $T_{0}=0.2, Q=1[1,1](\diamond)$ is also shown. The thin dashed line is a best fit to Eq. (3). Inset: $\Sigma$ vs $\dot{\gamma}$. 


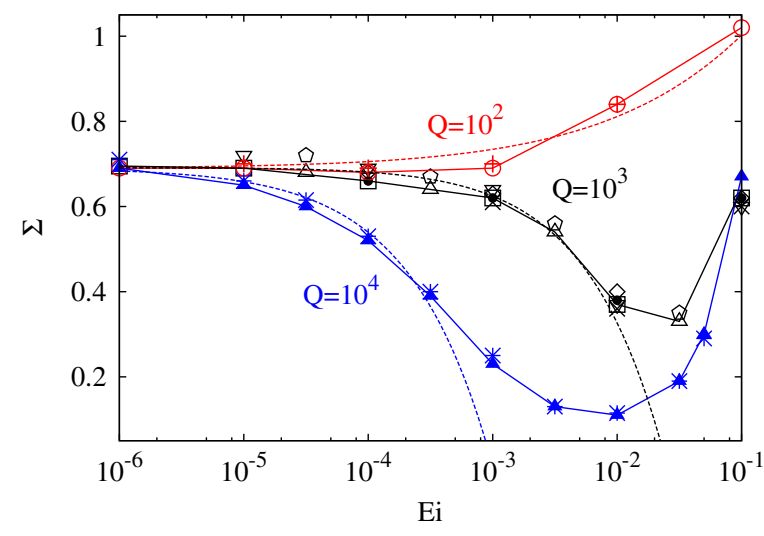

FIG. 2. Flow curves $\Sigma(Q$, Ei, 0$)$ of athermal underdamped systems and $\Sigma\left[Q^{\prime}, \mathrm{Ei}, T_{K}(Q, \mathrm{Ei})\right]$ of their thermostated counterparts (see text). Symbols are listed in Table I. Thin dashed lines are the best fit to Eq. (3), $\quad \Sigma=0.69+2 \sqrt{\mathrm{Ei}-}$ $0.17 T_{K}^{2 / 3} \ln \left(0.4 T_{K}^{5 / 6} / \mathrm{Ei}\right)^{2 / 3}$, where $T_{K}=0.15 Q \mathrm{Ei}$.

Perhaps less expectedly, we also find a narrowing of the overdamped regime with $T_{0}$, that is, the quality factor $Q_{c}\left(T_{0}\right)$ marking the departure from the scaling with Wi decreases with $T_{0}$ [our data suggest $Q_{c}\left(T_{0}=0\right) \approx 1$ whereas $Q_{c}\left(T_{0}=0.2\right)<1$ but do not allow for greater accuracy]. On rather general grounds, this can be explained by alluding to the excitation of higher-frequency modes at higher temperature, these modes having larger specific quality factors $Q$, or to the faster thermalization of the system (see below).

Inertial dynamics.-On increasing $Q$, past a small crossover region around $Q_{c}\left(T_{0}\right)$, one enters the underdamped regime, where the rheology is a priori described by the triplet $\left(Q, \mathrm{Ei}, T_{0}\right)$. What role does the inertial quality factor $Q$ play in that regime?

In fact, at low damping, $Q$ can no longer be interpreted as the number of not-too-damped inertial oscillations within a particle's cage. Indeed, localized excitations spread in the glass and, owing to nonlinearities, thermalize: their energy is redistributed across the whole vibrational spectrum. This process occurs over a time $\tau_{\text {th }}$ and expedites the decorrelation of the excitations when the velocity damping time $m / \zeta$ becomes longer than $\tau_{\text {th }}$. As a result, the velocity autocorrelation functions, which reflect single-particle dynamics, gain independence from $Q$, in the quiescent system at $T_{0}>0$ (in Fig. 1 of the Supplemental Material [22], we observe $\tau_{\text {th }} \approx 0.1 \tau_{\text {vib }}$ at $T_{0}=0.16$ ). Thus, one is lured into thinking that the underdamped rheology is insensitive to $Q$, in the same way as the equilibrium properties of liquids computed with MD are independent of the (weak) damping [18,23].

Contrary to this thought, the underdamped flow curves, plotted in Fig. 2, exhibit dramatic changes at large $Q$ (and low $T_{0}$ ), as they become nonmonotonic.

Clearly, the insensitivity to $Q$ was a fallacy. In fact, this parameter also controls energy dissipation in the system.
TABLE I. Parameters and symbols used in Figs. 2 and 3. Inertial quality factors are denoted $Q^{\prime}$, instead of $Q$, for the thermostated systems (see text).

\begin{tabular}{lccccc}
\hline \hline$Q$ & $Q^{\prime}$ & $\zeta$ & $m$ & $T_{0}$ & Symbol \\
\hline $10^{2}$ & & $10^{-2}$ & 1 & 0 & $\circ$ \\
& 10 & 0.1 & 1 & $T_{K}\left(Q=10^{2}, \mathrm{Ei}\right)$ & + \\
$10^{3}$ & & $10^{-3}$ & 1 & 0 & $\square$ \\
$10^{3}$ & & $10^{-2}$ & 100 & 0 & $\bullet$ \\
$10^{3}$ & & $3 \times 10^{-4}$ & 0.1 & 0 & $\triangle$ \\
& 1 & 1 & 1 & $T_{K}\left(Q=10^{3}, \mathrm{Ei}\right)$ & $\nabla$ \\
& 1 & 10 & 100 & $T_{K}\left(Q=10^{3}, \mathrm{Ei}\right)$ & $\diamond$ \\
& 1 & 0.3 & 0.1 & $T_{K}\left(Q=10^{3}, \mathrm{Ei}\right)$ & $\square$ \\
& 10 & 0.1 & 1 & $T_{K}\left(Q=10^{3}, \mathrm{Ei}\right)$ & $\times$ \\
$10^{4}$ & & $10^{-4}$ & 1 & 0 & $\mathbf{\Delta}$ \\
& 1 & 1 & 1 & $T_{K}\left(Q=10^{4}, \mathrm{Ei}\right)$ & $*$ \\
\hline \hline
\end{tabular}

When the damping is too weak compared to the energy input, the system heats up and strongly departs from thermal equilibrium with the heat reservoir at $T_{0}$. This is not a numerical artifact: in experiments on sheared granular matter, the "granular" temperature differs from room temperature [24]; temperature rises have also been borne out experimentally in shear bands in compressed BMG $[2,3]$ (incidentally, note that a negative rate dependence of the stress, known as "rate weakening," has also been reported in these materials [25]). For "dry" systems, heat is actually removed faster in simulations than in experiments, where its extraction must proceed through the boundaries [26]. Besides, nonmonotonic flow curves are not a marginal effect of the DPD thermostat; they were also observed by Salerno with a weak Langevin thermostat (see Fig. 2.3 of Ref. [27]).

Taking into account the heating of the sample, we propose to substitute, in the triplet $\left(Q, \mathrm{Ei}, T_{0}\right)$, the reservoir temperature $T_{0}$ with the actual kinetic temperature of the sample, $T_{K} \equiv(1 / 2 N) \sum_{i=1}^{N} m v_{i}^{2}$. To assess the contribution $T_{\dot{\gamma}}$ of the driving to $T_{K}$, we assume that the kinetic energy is mainly generated by plastic rearrangements, during which the elastic energy $(1 / 2 \rho) \Sigma_{0} \gamma_{y}$ per particle is first converted into kinetic energy and then gradually dissipated, over a time scale $\tau_{\text {damp }} \equiv m / \zeta$ in the underdamped regime. Thus, the density of simultaneous events is $m / \zeta \cdot \dot{\gamma} / \gamma_{y}$, and we arrive at

$$
T_{\dot{\gamma}} \approx\left(\frac{1}{2 \rho} \Sigma_{0} \gamma_{y}\right) \frac{m \dot{\gamma}}{\zeta \gamma_{y}} \approx \frac{\Sigma_{0}}{2 \rho} Q \text { Ei. }
$$

The scaling law with $Q \mathrm{Ei}$ is in very good agreement with the numerical data at $T_{0}=0$, as shown in Fig. 3, as long as $Q \gg 1$ (see Sec. V of Supplemental Material [22] for a tentative rationalization of the slight deviations). The predicted (0.29) and measured (0.15) prefactors differ by a factor of 2 precisely, probably because the released energy is actually equipartitioned between the kinetic 


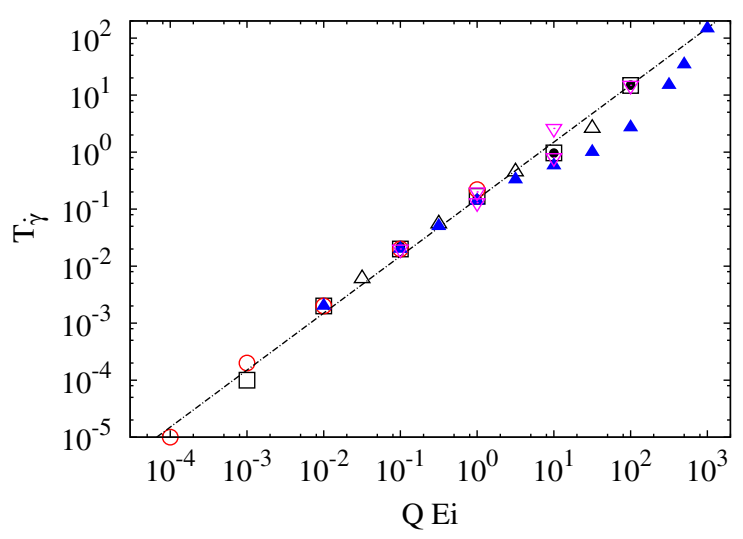

FIG. 3. Shear contribution $T_{\dot{\gamma}}=T_{K}-T_{0}$ to the kinetic temperature measured in underdamped samples vs $Q$ Ei. $(\nabla)$ Data at $T_{0}=0.2$. The line represents $T_{\dot{\gamma}}=0.15 Q \mathrm{Ei}$.

and elastic degrees of freedom, as for a harmonic oscillator. Furthermore, we observe a Boltzmann distribution, parametrized by $T_{\dot{\gamma}}$, of kinetic energies among the particles (Fig. 2 of the Supplemental Material [22]), which confirms the status of $T_{\dot{\gamma}}$ as the sample temperature. This is consistent with the "quasiequilibrium" situation (at $T_{K}$ ) reported by $\mathrm{Xu}$ et al. in strongly sheared athermal systems [28]. At finite $T_{0}$, we expect $T_{K} \approx T_{0}+T_{\dot{\gamma}}$, which is entirely compatible with our (limited) data set (Fig. 3).

Coming back to the underdamped flow curves, Fig. 2 gives ample evidence that the athermal flow curves at any $Q \gg Q_{c}(0)$ can be quantitatively reproduced by thermostating a less underdamped (but still inertial) system, at $Q^{\prime}<Q$, to the shear-rate-dependent temperature $T_{K}(Q, \mathrm{Ei})$ of the original system; this holds true at $T_{0}>0$ (data at $T_{0}=0.2$ not shown $)$. Put differently, $\Sigma\left(Q, \mathrm{Ei}, T_{0}\right)$ collapses onto a master curve $\tilde{\Sigma}\left(\mathrm{Ei}, T_{K}[Q, \mathrm{Ei})\right]$, irrespective of the value of $Q$. Thus, $Q$ does not impact the underdamped rheology as the inertial quality factor, but only via its control of $T_{K}(Q, \mathrm{Ei})$. It follows that inertial vibrations and thermal fluctuations have an analogous effect on the rheology: both are "agitation" forces that precipitate rearrangements, but the former increase with the shear rate, hence the severe rate weakening observed in strongly underdamped systems. We should mention that rate weakening is generally associated with a flow instability leading to shear banding $[29,30]$, but here we have not seen any banding of the velocity profiles. We believe that this is due to the rapidity of equilibration through thermal diffusion in small systems, which impedes the coexistence of bands sheared at different rates, thus (here) at distinct temperatures.

Chattoraj et al. [21], building on previous work by Johnson and Samwer [20], propounded the following formula for the temperature dependence of the stress,

$$
\Sigma\left(\dot{\gamma}, T_{0}\right)=\Sigma\left(\dot{\gamma}, T_{0}=0\right)-A T^{2 / 3} \ln \left(\frac{B T_{0}^{5 / 6}}{\dot{\gamma}}\right)^{2 / 3}
$$

where $A$ and $B$ are adjustable parameters. Substituting $T_{0}$ with $T_{K}=T_{0}+0.15 Q \mathrm{Ei}$ and $\dot{\gamma}$ with $\mathrm{Ei}$ in Eq. (3), we obtain predictions in broad agreement with our data, as shown in Fig. 2, as long as the flow remains underdamped and at low enough $T_{K}$.

These results do not imply that in underdamped systems inertia can be discarded in favor of temperature. Indeed, the collapse onto $\tilde{\Sigma}\left[\mathrm{Ei}, T_{K}(Q, \mathrm{Ei})\right]$ breaks down for $Q<Q_{c}\left(T_{0}\right)$, which highlights the operativeness of an inertial mechanism at $Q>Q_{c}\left(T_{0}\right)$, responsible, e.g., for the scaling of the inverse attempt frequency (multiplied by $\dot{\gamma}$ ) with $\mathrm{Ei}$, and not Wi. Still, it is noteworthy that the collapse holds down to values of $Q$ in the crossover region; in particular, systems at $Q=1$ display a macroscopic rheology close to the fully overdamped one at $T_{0}=0$, while a scan through their higher-temperature response gives access to the strongly underdamped rheology.

In summary, the variations of the macroscopic rheology of a model disordered solid with damping strength $\zeta$ (or particle mass $m$ ) can be collapsed into two flow regimes. When $Q \equiv \mathrm{Ei} /$ Wi is smaller than a threshold $Q_{c}\left(T_{0}\right)$, the system is overdamped. It is widely accepted that foams, concentrated emulsions, and dense colloidal suspensions belong in this regime. At fixed $T_{0}$, in particular $T_{0}=0$, the flow curves only depend on $\mathrm{Wi}$, which proves that the competition between the elastic interactions imposed by the PEL and dissipation forces dominates the rheology of these systems. This is compatible with the rheological models proposed by us and others in Refs. [10,11,31,32], but rules out all explanations based on the transverse sound velocity $c_{s}$ (which affects Ei and $Q$, but not $\mathrm{Wi}$ ).

Such explanatory scenarios based on $c_{s}$ could be valid in the (moderately) underdamped regime, at $Q \gtrsim Q_{c}\left(T_{0}\right)$. As a noteworthy example, Lemaître and Caroli [33] suggested the following scenario, later taken up and revised in [34,35]: avalanches of plastic events spread at speed $c_{s}$ and their spreading is limited by the driving, which generates independent plastic events. The ensuing incomplete plastic relaxation explains the increasing flow curve. However, the athermal MD system used in Ref. [33] appears similar to ours with $Q \approx 0.2<Q_{c}\left(T_{0}=0\right)$.

For even more strongly underdamped systems, at $Q \gg 10^{2}$, the flow curve becomes nonmonotonic at low bath temperature. Surprisingly, this transition has never been analyzed before, although the threshold for $Q$ does not seem unrealistically large: a crude estimate for a suspension of frictionless grains (of density $\rho$ and radius $a$ ) in a solvent of viscosity $\eta$ gives $Q \approx\left(0.1 a \sqrt{\rho \Sigma_{0}} / \eta\right)$. We showed that variations in the inertial properties of the material played no role per se in the transition; instead, the latter originates from the insufficient energy dissipation at large $Q$, which causes the sample to heat up (and hence, relax stress) all the more as the driving is fast, with the scaling law $T_{\dot{\gamma}} \propto Q \mathrm{Ei}$.

This rate-weakening mechanism persists until it is counterbalanced by the standard collisional increase of $\Sigma$ 
at high rates; this results in a minimum in the flow curve (see Sec. IV of Supplemental Material [22] for a discussion). The mechanism is reminiscent of the one producing a shear-banding instability in the soft glassy rheology variant proposed by Fielding et al. [36]. In soft glassy rheology, material subunits possess an (widely distributed) energy barrier for yielding, which decreases as the material is loaded. Yielding is then activated by an effective mechanical temperature $x$. In the variant of Ref. [36], $x$ is coupled to the local plastic activity and thus increases with the shear rate. In a similar fashion, in the shear transformation zone theory, the strain may localize via a coupling between the strain rate and the "configurational disorder temperature" [37]. The major conceptual divergence between these approaches and our observations in severely underdamped systems is the (effective or kinetic) nature of the temperature.

This difference echoes a vast debate in the metallic glass community regarding the origin of the softening of shear bands: does the band persist by softening because of heat production, hence, higher local temperatures, or, perhaps more probably, because of local configurational changes (in free volume or density), while the temperature rise is but a side effect $[2,3,26]$ ? Our findings do not contribute to settling this question, but they do certainly call for a clarification of the description of damping in rheological models.

We are grateful to Mark Robbins for first mentioning the nonmonotonic inertial flow curves, we acknowledge discussions with Kirsten Martens, Kamran Karimi, and Claus Heussinger. The simulations were carried out using the LAMMPS molecular dynamics software. J.-L. B. is supported by Institut Universitaire de France and by Grant No. ERC-2011-ADG20110209.

A. N. and J.R. contributed equally to this work.

*Present address: Centro Atómico Bariloche, 8400 S.C. Bariloche, Argentina. alexandre.nicolas@polytechnique.edu

[1] C. P. Amann, M. Ballauff, S. U. Egelhaaf, S. Fritschi, M. Fuchs, M. Krüger, M. Laurati, K. J. Mutch, K. Samwer, M. Siebenbürger et al., arXiv:1302.2030.

[2] J. Lewandowski and A. Greer, Nat. Mater. 5, 15 (2006).

[3] Y. Zhang, N. Stelmashenko, Z. Barber, W. Wang, J. Lewandowski, and A. Greer, J. Mater. Res. 22, 419 (2007).

[4] P. Schall, D. Weitz, and F. Spaepen, Science 318, 1895 (2007).

[5] C. E. Maloney and A. Lemaitre, Phys. Rev. E 74, 016118 (2006).

[6] J. Chattoraj, C. Caroli, and A. Lemaitre, Phys. Rev. E 84, 011501 (2011).

[7] V. Bulatov and A. Argon, Model. Simul. Mater. Sci. Eng. 2, 167 (1994).
[8] M. L. Falk and J. S. Langer, Phys. Rev. E 57, 7192 (1998).

[9] P. Sollich, F. Lequeux, P. Hébraud, and M. E. Cates, Phys. Rev. Lett. 78, 2020 (1997).

[10] P. Hébraud and F. Lequeux, Phys. Rev. Lett. 81, 2934 (1998).

[11] G. Picard, A. Ajdari, F. Lequeux, and L. Bocquet, Phys. Rev. E 71, 010501 (2005).

[12] M. Fuchs and M.E. Cates, Phys. Rev. Lett. 89, 248304 (2002).

[13] F. Puosi, J. Rottler, and J.-L. Barrat, Phys. Rev. E 89, 042302 (2014).

[14] A. Nicolas, F. Puosi, H. Mizuno, and J.-L. Barrat, J. Mech. Phys. Solids 78, 333 (2015).

[15] K. M. Salerno, C. E. Maloney, and M. O. Robbins, Phys. Rev. Lett. 109, 105703 (2012).

[16] K. M. Salerno and M. O. Robbins, Phys. Rev. E 88, 062206 (2013).

[17] C. P. C. Prado and Z. Olami, Phys. Rev. A 45, 665 (1992).

[18] T. Soddemann, B. Dünweg, and K. Kremer, Phys. Rev. E 68, 046702 (2003).

[19] R. A. Bagnold, Proc. R. Soc. A 225, 49 (1954).

[20] W. L. Johnson and K. Samwer, Phys. Rev. Lett. 95, 195501 (2005).

[21] J. Chattoraj, C. Caroli, and A. Lemaitre, Phys. Rev. Lett. 105, 266001 (2010).

[22] See Supplemental Material at http://link.aps.org/ supplemental/10.1103/PhysRevLett.116.058303 for more details.

[23] D. Evans and G. Morriss, Chem. Phys. 87, 451 (1984).

[24] W. Losert, L. Bocquet, T. C. Lubensky, and J. P. Gollub, Phys. Rev. Lett. 85, 1428 (2000).

[25] A. Dubach, F. D. Torre, and J. Löffler, Philos. Mag. Lett. 87, 695 (2007).

[26] N. P. Bailey, J. Schiøtz, and K. W. Jacobsen, Phys. Rev. B 73, 064108 (2006).

[27] K. Salerno, Ph.D. thesis, The Johns Hopkins University, 2013.

[28] N. Xu and C. S. O’Hern, Phys. Rev. Lett. 94, 055701 (2005).

[29] J. Yerushalmi, S. Katz, and R. Shinnar, Chem. Eng. Sci. 25, 1891 (1970).

[30] N. Spenley, X. Yuan, and M. Cates, J. Phys. II (France) 6, 551 (1996).

[31] A. Nicolas, K. Martens, and J.-L. Barrat, Europhys. Lett. 107, 44003 (2014).

[32] E. Agoritsas, E. Bertin, K. Martens, and J.-L. Barrat, Eur. Phys. J. E 38, 71 (2015).

[33] A. Lemaitre and C. Caroli, Phys. Rev. Lett. 103, 065501 (2009).

[34] C. Fusco, T. Albaret, and A. Tanguy, Eur. Phys. J. E 37, 43 (2014).

[35] J. Lin, E. Lerner, A. Rosso, and M. Wyart, Proc. Natl. Acad. Sci. U.S.A. 111, 14382 (2014).

[36] S. Fielding, M. Cates, and P. Sollich, Soft Matter 5, 2378 (2009).

[37] M. L. Manning, J. S. Langer, and J. M. Carlson, Phys. Rev. E 76, 056106 (2007). 